corresponds to the other ray tangent, and recalling Wilczynski's theorem, we are led at once to the final form in which we stated the geometric characterization of conjugate nets with equal point invariants. This characterization may of course be established analytically, and independently of Wilczynski's theorem, so that, on the basis of our general theorem, Wilczynski's characterization may then be obtained from ours.

HARVARD UNIVERSITY, August 22, 1917.

\title{
ON THE DIFFERENTIABILITY OF ASYMPTOTIC SERIES.
}

BY DR. J. F. RITT.

(Read before the American Mathematical Society, October 27, 1917.)

THE question of the differentiability of asymptotic series seems not to have received adequate treatment.* Writers on the theory of asymptotic convergence content themselves always with stating that if $P(x)$ has the asymptotic representation

$$
P(x) \sim a_{0}+a_{1} x+a_{2} x^{2}+\cdots+a_{n} x^{n}+\cdots,
$$

it may not have a derivative at all, and that even if a derivative does exist, the derivative may not admit of asymptotic development.

A failure to distinguish between the real and complex domains, in this connection, is responsible for a serious lacuna, which it is the purpose of this note to fill.

Let $P(x)$, defined in a sector with vertex at the origin, be analytic within the sector, in the neighborhood of the origin, and continuous on the sides of the sector, and at the origin. Let $P(x)$ have the asymptotic development of finite order

$$
P(x)=a_{0}+a_{1} x+a_{2} x^{2}+\cdots+a_{n} x^{n}+\epsilon(x) x^{n},
$$

where $n \geqq 2$ and where $\epsilon(x)$ goes to zero with $x$. We say that

* Since writing this note, I have ascertained by correspondence with Professor Birkhoff that he was familiar with and had used the result given here, but failed to publish it, being under the impression that it was contained in an article by W. B. Ford (Bull. de la Soc. Math. de France, 1911, p. 347). 
$P^{\prime}(x)$, the derivative of $P(x)$, which, of course, exists in the interior of the sector, exists also at the origin relative to any sector within the given one, and that in the inner sector $P^{\prime}(x)$ has the development

$$
P^{\prime}(x)=a_{1}+2 a_{2} x+3 a_{3} x^{2}+\cdots+(n-1) a_{n-1} x^{n-2}+\epsilon_{1}(x) x^{n-2} \text {. }
$$

The proof is simple. Put $x=1 / y$. Then $P(1 / y)$ is defined and is analytic, for sufficiently large values of $y$, in a sector $S$, which is very simply related to the sector of definition of $P(x)$, and has the asymptotic development

We have

$$
P(1 / y)=a_{0}+\frac{a_{1}}{y}+\frac{a_{2}}{y^{2}}+\cdots+\frac{a_{n}}{y^{n}}+\frac{\epsilon(1 / y)}{y^{n}} .
$$

$$
\frac{d}{d y} P(1 / y)=-\frac{a_{1}}{y^{2}}-\frac{2 a_{2}}{y^{3}}-\cdots-\frac{n a_{n}}{y^{n+1}}-\frac{n \epsilon(1 / y)}{y^{n+1}}+\frac{1}{y^{n}} \frac{d}{d y} \epsilon\left(\frac{1}{y}\right) .
$$

Consider now any sector $S^{\prime}$ interior to $S$, the sector of definition of $P(1 / y)$. For sufficiently large values of $y$ any point of $S^{\prime}$ is at least at a distance of unity from the sides of $S$. For any such point in $S^{\prime}$, we have

$$
\frac{d}{d y} \epsilon(1 / y)=\frac{1}{2 \pi i} \int_{o} \frac{\epsilon(1 / \zeta) d \zeta}{(\zeta-y)^{2}},
$$

the integration being made around a circle of radius unity, so that

$$
\left|\frac{d}{d y} \epsilon(1 / y)\right|<M,
$$

where $M$ is the maximum modulus of $\epsilon(1 / y)$ along the circle. Since $\epsilon(1 / y)$ goes to zero as $y$ becomes infinite, it is clear that we may collect the last three terms in the expression above for the derivative of $P(1 / y)$, and write

$$
\frac{d}{d y} P(1 / y)=-\frac{a_{1}}{y^{2}}-\frac{2 a_{2}}{y^{3}}-\cdots-\frac{(n-1) a_{n-1}}{y^{n}}-\frac{\epsilon_{1}(1 / y)}{y^{n}},
$$

where $\epsilon_{1}(1 / y)$ goes to zero with $1 / y$.

Reverting now to $P(x)$,

$$
\begin{aligned}
P^{\prime}(x) & =\frac{d}{d y} P(1 / y) \frac{d y}{d x}=-y^{2} \frac{d}{d y} P(1 / y) \\
& =a_{1}+\frac{2 a_{2}}{y}+\cdots+\frac{(n-1) a_{n-1}}{y^{n-2}}+\frac{\epsilon_{1}(1 / y)}{y^{n-2}},
\end{aligned}
$$


and finally

$$
P^{\prime}(x)=a_{1}+2 a_{2} x+\cdots+(n-1) a_{n-1} x^{n-2}+\epsilon_{1}(x) x^{n-2},
$$

which is the asymptotic development sought. Also it results easily from the fact that $P^{\prime}(x)$ approaches $a_{1}$ as $x$ goes to zero that the derivative at the origin exists and is equal to $a_{1}$.

It is interesting to notice that a single differentiation has lost us two terms of the development of $P(x)$ * $^{*}$ However, if $P(x)$ has an infinite asymptotic development, it is clear that $P^{\prime}(x)$ will also have an infinite development, and in fact that the development of $P(x)$ can be differentiated formally any number of times.

The above proof applies, of course, to other domains than sectors; for instance, we might use the horn angle obtained by an inversion relative to the origin, and a reflection across the real axis of the infinite strip between any two parallel lines.

Lastly, it is important to notice that we have also established above the differentiability in the complex domain of asymptotic developments in descending powers of the variable.

Columbia University.

\section{DARBOUX'S CONTRIBUTION TO GEOMETRY.}

BY PROFESSOR L. P. EISENHART.

(Read before the joint session of the American Mathematical Society and the Mathematical Association of America at Cleveland, Ohio, September 6,1917 .)

Gaston Darboux was born in 1842 at Nîmes, a place of interest to mathematicians because here from 1819 to 1831 Gergonne edited his Annales, and incidentally exerted a great influence on the development of geometry. At the age of eighteen Darboux went to Paris, in whose intellectual life he had a prominent part for fifty-seven years. As a student, first at the Ecole Polytechnique and then at the Ecole Normale, his unusual mathematical ability made him conspicuous. His

* The loss of the term $n a_{n} x^{n-1}$ is only apparent. This can be shown by taking the radius of the circle of integration above equal to $k|y|$, where $k$ is some number independent of $y$. I prefer the proof above because of its applicability to more general domains than sectors. 